\title{
ECONOMICS
}

\section{Cooperation from competition}

Sci. Adv. 4, eaat2201 (2018)

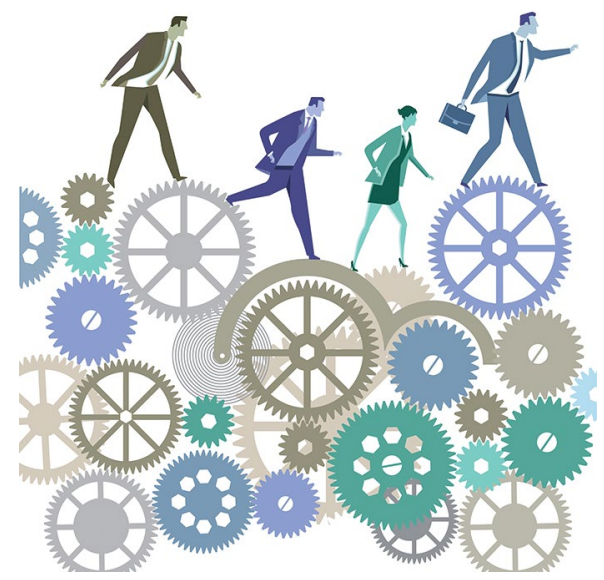

Credit: scusi/Alamy Stock Vector

Prosocial behaviour, like trust and cooperation, is a fundamental feature of human society. Despite its ubiquity and importance, there is no scientific consensus about the origins of prosociality.

A new paper by Patrick Francois, of the University of British Columbia, and colleagues presents contemporary evidence that prosocial behaviour evolved as a result of group-level competition dynamics. When faced with a competitive environment, groups that are internally cooperative are able to compete more effectively.

Competition selects for prosocial behaviour by weeding out less cooperative groups and encouraging the imitation of the norms of more successful groups. The authors present survey evidence showing that people who work in more competitive industries, who live in areas that experience an increase in competition and who switch to working in more competitive sectors all express higher levels of trust in others. They also show experimentally that participants in more competitive conditions contribute larger amounts to public goods, increase contributions following a loss to a more cooperative competitor and also express higher levels of trust in others.

Rather than promoting purely cut-throat behaviour, competition can encourage the spread of prosocial norms. The work highlights the potential importance of future studies that trace the long-term effects of competitive environments on human cooperation.

\section{Aisha Bradshaw}

Published online: 18 October 2018

https://doi.org/10.1038/s41562-018-0464-7 